\title{
400.
}

\section{ON THE CUBIC CURVES INSCRIBED IN A GIVEN PENCIL OF SIX LINES.}

[From the Quarterly Journal of Pure and Applied Mathematics, vol. Ix. (1868), pp. $210-221$.

WE have to consider a pencil of six lines, that is, six lines meeting in a point, and a cubic curve touching each of the six lines. As a cubic curve may be made to satisfy nine conditions, the cubic curve will involve three arbitrary parameters; but if we have any particular curve touching the six lines, then transforming the whole figure homologously, the centre of the pencil being the pole and any line whatever the axis of homology, the pencil of lines remains unaltered, and the new curve touches the six lines of the pencil; the transformation introduces three arbitrary constants, and the general solution is thus given as such homologous transformation of a particular solution. To show the same thing analytically, take $(x=0, y=0, z=0)$ for the axes of coordinates, the lines $x=0, y=0$ being any two lines through the centre of the pencil, so that the equation of the pencil is $(* \gamma x, y)^{6}=0$, then if $\phi(x, y, z)=0$ is the equation of a cubic curve touching the six lines, the equation of the general curve touching. the six lines will be $\phi(x, y, \alpha x+\beta y+\gamma z)=0$; or what is the same thing, considering the coordinate $z$ as implicitly containing three arbitrary constants, viz. an arbitrary multiplier and the two arbitrary parameters of the line $z=0$, then the equation $\phi(x, y, z)=0$ may be taken to be that of the cubic touching the six lines.

Now the given binary sextic $(* \gamma x, y)^{6}$ may be expressed in the form $P^{2}+Q^{3}$, where $P$ is a cubic function, $Q$ a quadric function, of the coordinates $(x, y)$; or, what is the same thing, but introducing for homogeneity a constant $c$, we may write

$$
\left(* \gamma(x, y)^{6}=c\left[\left(a, h, k, b_{\chi} \chi x, y\right)^{3}\right]^{2}+4\left[(j, l, f \gamma x, y)^{2}\right]^{3} ;\right.
$$

C. VI. 
in fact, comparing the two sides of this equation, we have each of the seven coefficients of the sextic equal to a function of the seven quantities $a \sqrt{ }(c), h \sqrt{ }(c), k \sqrt{ }(c)$, $b \sqrt{ }(c), j, l, f$; so that conversely, these seven quantities are determinable (not however rationally) in terms of the coefficients of the given sextic. And when the sextic is expressed in the foregoing form, then it will presently be shown that we have

$$
\left(a, h, k, b \gamma(x, y)^{3}+3 z\left(j, l, f \gamma(x, y)^{2}+c z^{3}=0,\right.\right.
$$

or, what is the same thing,

$$
\left(a, b, c, f, 0, h, 0, j, k \gamma(x, y, z)^{3}=0,\right.
$$

as the equation of a cubic curve touching the six given lines; and by what precedes, it appears that this may be taken to be the equation of the general cubic curve which touches the six given lines. On account of the arbitrary constant $c$, it is sufficient to replace $z$ by $\alpha x+\beta y+z$, or, what is the same thing, to consider $z=0$ as the equation of an arbitrary line, but without introducing therein an arbitrary multiplier.

To sustain the foregoing result, consider the cubic

$$
\left(a, b, c, f, g, h, i, j, k, l \gamma(x, y, z)^{3}=0,\right.
$$

then in general if $A=\left(, \gamma(x, y, z)^{3}, B=\left(, \gamma(x, y, z)^{2}(\alpha, \beta, \gamma), C=(\right.\right.$, $\chi x, y, z)(\alpha, \beta, \gamma)^{2}$, $D=(,, \chi \alpha, \beta, \gamma)^{3}$, the equation of the pencil of tangents drawn from the point $(\alpha, \beta, \gamma)$ to the curve is

$$
A^{2} D^{2}-6 A B C D+4 A C^{3}+4 B^{3} D-3 B^{2} C^{2}=0,
$$

but writing for shortness

so that

$$
(,, \chi x, y, z)^{3}=\left(A^{\prime}, B^{\prime}, C^{\prime}, D^{\prime} \chi 1, z\right)^{3},
$$

$$
\begin{array}{lc}
A^{\prime}=\left(a, h, k, b \gamma(x, y)^{3},\right. \\
B^{\prime}=\left(j, l, f \gamma(x, y)^{2},\right. \\
C^{\prime}= & (g, i \gamma(x, y), \\
D^{\prime}= & c
\end{array}
$$

then for the tangents from the point $(x=0, y=0)$, writing $(\alpha, \beta, \gamma)=(0,0,1)$, we have

$$
\begin{array}{cc}
A=\left(A^{\prime}, B^{\prime}, C^{\prime}, D^{\prime} \chi 1, z\right)^{3}, \\
B= & \left(B^{\prime}, C^{\prime}, D^{\prime} \chi 1, z\right)^{2}, \\
C= & \left(C^{\prime}, D^{\prime} \backslash 1, z\right), \\
D= & D^{\prime},
\end{array}
$$

and thence the equation of the pencil of tangents is

$$
A^{\prime 2} D^{\prime 2}-6 A^{\prime} B^{\prime} C^{\prime} D^{\prime}+4 A^{\prime} C^{\prime 3}+4 B^{\prime 3} D^{\prime}-3 B^{\prime 2} C^{\prime 2}=0 .
$$

Hence for the curve

$$
\left(a, b, c, f, 0, h, 0, j, k, l \gamma(x, y)^{3}=0,\right.
$$


we have $g=0, i=0$, and therefore $C^{\prime}=0$; the equation of the pencil of tangents is $A^{\prime 2} D^{\prime 2}+4 B^{\prime 3} D^{\prime}=0$, or throwing out the constant factor $D^{\prime}$, and then replacing $A^{\prime}, B^{\prime}, D^{\prime}$ by their values, the equation of the pencil of tangents is

$$
c\left[\left(a, h, k, b \gamma(x, y)^{3}\right]^{2}+4\left[\left(j, l, f_{\zeta}(x, y)^{2}\right]^{3}=0,\right.\right.
$$

which is the before-mentioned result.

The coefficients $a \sqrt{ }(c), h \sqrt{ }(c), k \sqrt{ }(c), b \sqrt{ }(c), j, l, f$, or (as we may call them) the coefficients of the cubic curve, are, it has been seen, functions of the coefficients of the given sextic $\left(* \gamma(x, y)^{6}\right.$; hence the invariants $S$ and $T$ of the cubic curve are also functions of the coefficients of the sextic, and it is easy to see that they are in fact invariants (not however rational invariants) of the sextic. To verify this, it is only necessary to show that the invariants $S$ and $T$ are functions of the invariants of the functions $\sqrt{ }(c) .\left(a, h, k, b \chi(x, y)^{3}\right.$ and $(j, l, f \chi x, y)^{2}$; for if this be so, they will be invariants of the function

$$
\left[c\left(a, h, k, b \gamma(x, y)^{3}\right]^{2}+4\left[\left(j, l, f \gamma(x, y)^{2}\right]^{3},\right.\right.
$$

that is of the sextic. We have in fact the general theorem, that if $P, Q, R, \ldots$ be any quantics in $(x, y, \ldots)$, and $\phi(P, Q, R, \ldots)$ a function of these quantics, homogeneous in regard to $(x, y, \ldots)$, then any function of the coefficients of $\phi$, which is an invariant of the quantics $P, Q, R, \ldots$ is also an invariant of $\phi$.

Considering for greater convenience the function

$$
\left(a, h, k, b \gamma(x, y)^{3}\right.
$$

in place of $\sqrt{ }(c) .\left(a, h, k, b \gamma(x, y)^{3}\right.$, the invariants of the two functions $\left(a, h, k, b \gamma(x, y)^{3}\right.$ and $\left(j, l, f \gamma(x, y)^{2}\right.$ are as follows:

$$
\begin{aligned}
\square= & a^{2} b^{2}-6 a b h k+4 a k^{3}+4 b h^{3}-3 h^{2} k^{2} \\
\nabla= & f j-l^{2} \\
\Theta= & j\left(b h-k^{2}\right)+l(h k-a b)+f\left(a k-h^{2}\right) \\
R= & +1 a^{2} f^{3} \\
& +6 a b f l j \\
& -6 a h f^{2} l \\
& -6 a k f^{2} j \\
& +12 a k f l^{2} \\
& +1 b^{2} j^{3} \\
& -6 b h f j^{2} \\
& +12 b h j l^{2} \\
& -6 b k j^{2} l \\
& +9 h^{2} f^{2} j \\
& -18 h k f j l \\
& +9 k^{2} f^{2} \\
& -8 a b l^{3}
\end{aligned}
$$


viz. $\square, \nabla$ are the discriminants of the two functions respectively, and $\Theta, R$ are simultaneous invariants of the two functions, $R$ being in fact the resultant. The corresponding invariants of the functions $\sqrt{ }(c) \cdot\left(a, h, k, b \gamma(x, y)^{3}\right.$, and $(j, l, f \gamma x, y)^{2}$ are obviously $c^{2} \square, \nabla, c \Theta$ and $c R$.

The values of $S$ and $T$ are obtained from the Tables 62 and 63 of my "Third Memoir on Quantics," Phil. Trans. vol. CxLvi. (1856), pp. 627-647, [144], by merely writing therein $g=i=0$. It appears that they are in fact functions of $c^{2} \square, \nabla, c \Theta$ and $c R$; viz. we have

$$
\begin{aligned}
& S=\nabla^{2}+\imath \Theta, \\
& T=8 \nabla^{3}+c(4 R+12 \nabla \Theta)+c^{2} \square .
\end{aligned}
$$

The invariants of the sextic $(* \gamma x, y)^{6}$, if for a moment the coefficients of this sextic are taken to be $(a, b, c, d, e, f, g)$, that is, if the sextic be represented by $(a, b, c, d, e, f, g \gamma x, y)^{6}$ are the quadrinvariant $\left(=a g-6 b f+15 c e-10 d^{2}\right)$, Table No. 31 and Salmon's A., p. 203(1), the quartinvariant, No. 34, and Salmon's B., p. 203, the sextinvariant No. 35, and Salmon's C., p. 204, and the discriminant, which is a function of the tenth order $=a^{5} g^{5}+\& c$. recently calculated for the general form, Salmon, pp. 205-207, say these invariants are $Q_{2}, Q_{4}, Q_{6}$ and $Q_{10}$. These several invariants are functions of the above-mentioned expressions $c^{2} \square, \nabla, c \Theta$ and $c R$; whence, conversely, these quantities are functions of the four invariants $Q_{2}, Q_{4}, Q_{6}, Q_{10}$; and the invariants $S, T$ of the cubic curve, being functions of $c^{2} \square, \nabla, c \Theta$ and $c R$, are also, as they should be, functions of the invariants $Q_{2}, Q_{4}, Q_{6}$ and $Q_{10}$ of the sextic pencil $(* \gamma x, y)^{6}$.

To effect the calculation of $Q_{2}, Q_{4}$ and $Q_{6}$, I remark that inasmuch as by a linear transformation, the quadric $\left(j, l, f \gamma(x, y)^{2}\right.$ may be reduced to the form $2 l x y$, and that the invariants of $(a, h, k, b \gamma x, y)^{3}$ and $2 l x y$ are

$$
\begin{aligned}
\square & =a^{2} b^{2}-6 a b h k+4 a k^{3}+4 b h^{2}-3 h^{2} k^{2}, \\
\nabla & =-l^{2}, \\
\Theta & =-l(a b-h k), \\
R & =-8 l^{3} a b,
\end{aligned}
$$

hence, writing $j=0, f=0$, and writing also $c=1$, we may consider the sextic

that is

$$
\left[(a, h, k, b \gamma x, y)^{3}\right]^{2}+32 l^{3} x^{3} y^{3},
$$

$$
\left(a^{2}, a h, \frac{1}{5}\left(2 a k+3 h^{2}\right), \frac{1}{10}\left(a b+9 h k+16 l^{3}\right), \frac{1}{5}\left(2 b h+3 k^{2}\right), b k, b^{2} \gamma x, y\right)^{6},
$$

the invariants whereof are found to be functions of the last mentioned values of $\square, \nabla, \Theta, R$; to pass to the given sextic $(* \chi x, y)^{6}$, put equal to

$$
c\left[\left(a, h, k, b \gamma(x, y)^{3}\right]^{2}+4\left[(j, l, f \gamma x, y)^{2}\right]^{3},\right.
$$

we have only to consider $\square, \nabla, \Theta, R$ as having their before-mentioned general values, and to restore the coefficient $c$ by the principle of homogeneity.

1 The pages refer to Salmon's Lessons Introductory to the Modern Higher Algebra (Second Edition, 1866). In the Fourth Edition, 1885, the values are given, pp. 260-265. 
As regards the discriminant $Q_{10}$, this as already remarked, has been calculated for the general form, but for the present purpose it is easier, by dealing directly with the form $\left[\left(a, h, k, b \gamma(x, y)^{3}\right]^{2}+32 l^{3} x^{3} y^{3}\right.$, and then interpreting $\square, \nabla, \Theta, R$ and restoring the coefficient $c$ as above, to obtain the discriminant $Q_{10}$ of the function

$$
\left[c\left(a, h, k, b \gamma(x, y)^{3}\right]^{2}+4\left[(j, l, f \gamma x, y)^{2}\right]^{3}\right.
$$

in the required form, as a function of $c^{2} \square, \nabla, c \Theta, c R$.

I find after some laborious calculations

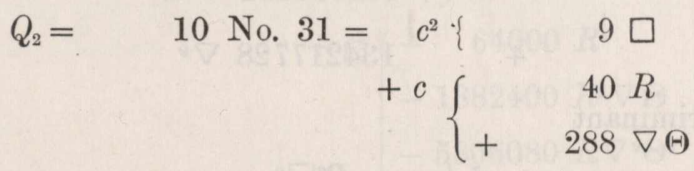

$$
\begin{aligned}
& +\left\{256 \nabla^{3}\right. \\
& Q_{4}=10000 \text { No. } 34=c^{4}\left\{-\quad 99 \square \square^{2}\right. \\
& +c^{3}\left\{\begin{array}{l}
-\quad 400 R \square \\
+\quad 2304 \nabla \Theta \square \\
+\quad 8640 \Theta^{3}
\end{array}\right. \\
& +c^{2}\left\{\begin{array}{r}
12800 R \nabla \Theta \\
+\quad 82944 \nabla^{2} \Theta^{2} \\
+\quad 4608 \nabla^{3} \square
\end{array}\right. \\
& +c\left\{\begin{array}{r}
20480 R \nabla^{3} \\
+147456 \nabla^{4} \Theta
\end{array}\right. \\
& +\quad 65536 \nabla^{6} \\
& Q_{6}=1000000 \text { No. } 35=c^{6}\left\{+\quad 7992 \square^{3}\right. \\
& +c^{5}\left\{\begin{array}{lc}
+ & 72000 R \square^{2} \\
+ & 145152 \nabla \Theta^{2} \square \\
- & 622080 \Theta^{3} \square
\end{array}\right. \\
& +c^{4}\left\{\begin{array}{c}
160000 R^{2} \square \\
+\quad 691200 R \nabla \Theta \square \\
+\quad 3456000 R \Theta^{3} \\
+\quad 3815424 \nabla^{2} \Theta^{2} \square \\
+\quad 36080640 \nabla \Theta^{4} \\
+\quad 635904 \nabla^{3} \square^{2}
\end{array}\right. \\
& +c^{3}\left(+33177600 R \nabla^{2} \Theta^{2}\right. \\
& +\quad 4669440 R \nabla^{3} \\
& +217645056 \nabla^{3} \Theta^{3} \\
& +23003136 \nabla^{4} \Theta \square
\end{aligned}
$$




$$
\begin{aligned}
& +c^{2}\left\{\begin{array}{l}
+\quad 8192000 R^{2} \nabla^{3} \\
+110100480 R \nabla^{4} \Theta \\
+509607936 \nabla^{5} \Theta^{2} \\
+\quad 14155776 \nabla^{6} \square
\end{array}\right. \\
& +c\left\{\begin{array}{r}
62914560 R \nabla^{6} \\
+452984832 \nabla^{7} \Theta
\end{array}\right. \\
& +\quad 134217728 \nabla^{9}
\end{aligned}
$$

$Q_{10}=$ multiple of discriminant

$$
\begin{aligned}
& =c^{7}\left\{-\quad R^{3} \square^{2}\right. \\
& +c^{6}\left(-8 R^{4} \square\right. \\
& \left\{-24 R^{3} \nabla \Theta \square\right. \\
& \left(+64 R^{3} \Theta^{3}\right. \\
& +c^{5}\left(-16 R^{5}\right. \\
& -96 R^{4} \nabla \Theta \\
& +48 R^{4} \nabla^{2} \Theta^{2} \\
& \left(-16 R^{4} \nabla^{3} \square\right. \\
& +c^{4}\left\{-64 R^{4} \nabla^{3}\right. \text {, }
\end{aligned}
$$

to which may be joined

$$
\begin{aligned}
& Q_{2}{ }^{2}=c^{4}\left\{\quad 81 \square^{2}\right. \\
& +c^{3}\left\{\begin{array}{r}
720 R \square \\
+\quad 5184 \nabla \Theta \square
\end{array}\right. \\
& +c^{2}\left\{\begin{array}{c}
1600 R^{2} \\
+\quad 23040 R \nabla \Theta \\
+\quad 82944 \nabla^{2} \Theta^{2} \\
+\quad 4608 \nabla^{3} \square
\end{array}\right. \\
& +c\left\{\begin{array}{r}
20480 R \nabla^{3} \\
+147456 \nabla^{4} \Theta
\end{array}\right. \\
& +\quad 65536 \square^{6}
\end{aligned}
$$

$$
\begin{aligned}
& Q_{2}{ }^{2}-Q_{4}=c^{4}\left\{\quad 180 \square^{2}\right. \\
& +c^{3}\left\{\begin{aligned}
1120 R \square \\
+\quad 2880 \nabla \Theta \square \\
-8640 \Theta^{3}
\end{aligned}\right. \\
& +c^{2}\left\{\begin{array}{c}
1600 R^{2} \\
+10240 R \nabla \Theta
\end{array}\right.
\end{aligned}
$$

and

$$
\begin{aligned}
\frac{1}{8}\left(Q_{6}-8 Q_{2}{ }^{3}\right)= & c^{6}\left\{\begin{array} { c } 
{ 2 7 0 \square ^ { 3 } } \\
{ + c ^ { 5 } }
\end{array} \left\{\begin{array}{cc}
-\quad 720 R \square^{2} \\
-\quad 51840 \nabla \Theta \square^{2} \\
-\quad 77760 \Theta^{3} \square
\end{array}\right.\right.
\end{aligned}
$$




$$
\begin{aligned}
& +c^{4}\left\{\begin{array}{l}
-\quad 23200 R^{2} \square \\
-\quad 535680 R \Theta \nabla \square \\
+\quad 432000 R \Theta^{3} \\
-1762560 \nabla^{2} \Theta^{2} \square \\
+4510080 \nabla \Theta^{4} \\
+\quad 17280 \nabla^{3} \square^{2}
\end{array}\right. \\
& +c^{3}\left\{\begin{array}{l}
-\quad 64000 R^{3} \\
-1382400 R^{2} \nabla^{-} \Theta \\
-5806080 R \nabla^{2} \Theta^{2} \\
+\quad 30720 R \nabla^{3} \square \\
+3317760 \nabla^{3} \Theta^{3} \\
-1105920 \nabla^{4} \Theta \square
\end{array}\right. \\
& +c^{2}\left\{\begin{array}{l}
-204800 R^{2} \nabla^{3} \\
-3932160 R \nabla^{4} \Theta .
\end{array}\right.
\end{aligned}
$$

The foregoing values of $S$ and $T$ give

$$
\begin{aligned}
& T^{2}-64 S^{3}=c^{4}\left\{\quad \square{ }^{2}\right. \\
& +c^{3}\left\{\begin{array}{c}
8 R \square \\
+24 \nabla \Theta \square \\
-64 \Theta^{3}
\end{array}\right. \\
& +c^{2}\left\{\begin{array}{c}
16 R^{2} \\
+96 R \nabla \Theta \\
-48 \nabla^{2} \Theta^{4} \\
+16 \nabla^{3} \square
\end{array}\right. \\
& +c \quad 64 R \nabla^{3} \text {, }
\end{aligned}
$$

so that

$$
\begin{aligned}
Q_{10} & =-c^{3} R^{3}\left(T^{2}-64 S^{3}\right), \\
64 S^{3}-T^{2} & =\frac{Q_{10}}{c^{3} R^{3}} \\
S & =\nabla^{2}+c \Theta,
\end{aligned}
$$

and therefore

$$
64-\frac{T^{2}}{S^{3}}=\frac{Q_{10}}{c^{3} R^{3}\left(\nabla^{2}+c \Theta\right)^{3}}=\frac{Q_{10}}{\left\{c R\left(\nabla^{2}+c \Theta\right)\right\}^{3}},
$$

formulæ which are interesting in the theory. 
We have

$$
\begin{aligned}
& c \Theta=S-\nabla^{2}, \\
& c^{2} \square=T-12 \nabla S+4 \nabla^{3}-4 c R,
\end{aligned}
$$

and if by means of these values we eliminate $c \Theta$ and $c^{2} \square$, we obtain $Q_{2}, Q_{4}, Q_{6}$ and $Q_{10}$ as functions of $S, T, \nabla$ and $c R$. Choosing instead of $Q_{4}$ and $Q_{6}$ the combinations $Q_{2}{ }^{2}-Q_{4}$ and $Q_{6}-8 Q_{2}{ }^{3}$, and forming also the expression for the combination $Q_{2}\left(Q_{2}{ }^{2}-Q_{4}\right)$, we have thus the system of formulæ

$$
\begin{aligned}
Q_{2}= & \quad 9 T+180 \nabla S+4 \nabla^{3}+4 c R \\
\frac{1}{20}\left(Q_{2}{ }^{2}-Q_{4}\right)= & +9 T^{2} \\
& -432 S^{3} \\
& -72 T \nabla S \\
& -72 T \nabla^{3} \\
& -16 T c R \\
& +864 \nabla^{2} S^{2} \\
& +144 \nabla \nabla^{4} S \\
& +128 \nabla S c R
\end{aligned}
$$

$\frac{1}{80}\left(Q_{6}-8 Q_{2}{ }^{3}\right)=$

$$
\begin{aligned}
& +\quad 27 T^{3} \\
& \text { - } 4212 T^{2} \nabla S \\
& +\quad 6588 T^{2} \nabla^{3} \\
& +\quad 252 T^{2} c R \\
& \text { - } 7776 \text { TS }^{3} \\
& \text { - } 16848 T \nabla^{2} S^{3} \\
& +\quad 3456 T \nabla{ }^{4} S \\
& \text { - } 2592 T \nabla S c R \\
& \text { - } 1296 T \nabla^{6} \\
& \text { - } \quad 1824 T \nabla{ }^{3} c R \\
& \text { - } \quad 448 T c^{2} R^{2} \\
& +544320 \nabla S^{4} \\
& -461376 \nabla^{3} S^{3} \\
& +\quad 74304 c R S^{3} \\
& +15552 \nabla{ }^{5} S^{2} \\
& \text { - } 10368 \nabla^{2} S^{2} c R \\
& \text { - } 10728 \nabla^{7} S \\
& \text { - } \quad 3264 \nabla{ }^{4} S c R \\
& \text { - } \quad 1536 \nabla S c^{2} R^{2} \text {, }
\end{aligned}
$$

$+\quad 81 T^{3}$

$+\quad 972 T^{2} \nabla S$

- $612 T^{2} \nabla^{3}$

- $\quad 108 T^{2} c R$

- $\quad 3888 T S^{3}$

- $5184 T \nabla^{2} S^{3}$

$-11952 T \nabla{ }^{4} S$

- $2016 T \nabla S c R$

- $\quad 288 T \nabla^{6}$

- $\quad 352 T \nabla{ }^{3} c R$

- $\quad 64 T c^{2} R^{2}$

- $77760 \nabla S^{4}$

$+153792 \nabla^{3} S^{3}$

- $1728 c R S^{3}$

$+29376 \nabla^{5} S^{2}$

$+26996 \nabla^{2} S^{2} c R$

$+\quad 576 \nabla^{7} S$

$+\quad 1088 \nabla{ }^{4} S c R$

+ $512 \nabla S c^{2} R^{2}$, 
and, as mentioned above,

$$
Q_{10}=c^{3} R^{3}\left(-T^{2}+64 S^{3}\right) .
$$

The just-mentioned value of $Q_{10}$ should, I think, admit of being established a priori, and if this be so, then the substitution of the values of $S$ and $T$ in terms of $c^{2} \square$, $\nabla, c \Theta, c R$, would be the easiest way of arriving at the before-mentioned expression of $Q_{10}$ in terms of these same quantities. The calculation by which this expression was arrived at, is however not without interest, and it will be as well to indicate the mode in which it was effected.

Calculation of $Q_{10}$.

We have to find the discriminant of

$$
c\left[\left(a, h, k, b \gamma(x, y)^{3}\right]^{2}+32 l^{3} x^{3} y^{3} .\right.
$$

Consider for a moment the more general form $P^{2}+4 Q^{3}$, then to find the discriminant, we have to eliminate between the equations

$$
\begin{aligned}
& P \frac{d P}{d x}+6 Q^{2} \frac{d Q}{d x}=0 \\
& P \frac{d P}{d y}+6 Q^{2} \frac{d Q}{d y}=0
\end{aligned}
$$

these are satisfied by the system $P=0, Q^{2}=0$, and it follows that if $R$ be the resultant of the equations $P=0, Q=0$, then the discriminant in question contains the factor $R^{2}$. For the other factor we may reduce the system to

$$
\begin{array}{r}
P \frac{d P}{d x}+6 Q^{2} \frac{d Q}{d x}=0 \\
\frac{d P}{d x} \frac{d Q}{d y}-\frac{d P}{d y} \frac{d Q}{d x}=0
\end{array}
$$

Now writing $Q=2 l x y$, these equations become

$$
\begin{aligned}
& P \frac{d P}{d x}+48 l^{3} x^{2} y^{3}=0 \\
& l\left(x \frac{d P}{d x}-y \frac{d P}{d y}\right)=0
\end{aligned}
$$

the resultant of which is $=l^{3}$ into resultant of the system

$$
\begin{aligned}
& P \frac{d P}{d x}+48 l^{3} x^{2} y^{3}=0 \\
& x \frac{d P}{d x}-y \frac{d P}{d y}=0
\end{aligned}
$$

C. VI. 
but in virtue of the second equation, we have

$$
P=\frac{1}{3}\left(x \frac{d P}{d x}+y \frac{d P}{d y}\right)=\frac{2}{3} y \frac{d P}{d y}
$$

which reduces the first equation to

$$
2 y \frac{d P}{d x} \frac{d P}{d y}+144 l^{3} x^{2} y^{3}=0
$$

or omitting the factor $2 y$, to

$$
\frac{d P}{d x} \frac{d P}{d y}+72 l^{3} x^{2} y^{3}=0
$$

Hence, writing $P=\sqrt{ }(c) \cdot\left(a, h, k, b_{\chi} x, y\right)^{3}$, and therefore $\frac{d P}{d x}=3 \sqrt{ }(c) \cdot\left(a, h, k \gamma(x, y)^{2}\right.$, $\frac{d P}{d y}=3 \sqrt{ }(c) \cdot\left(h, k, b \gamma(x, y)^{2} ;\right.$ writing also $y=1$, the two equations become

$$
\begin{gathered}
c\left(a, h, k_{\chi} x, 1\right)^{2}\left(h, k, b_{\chi} x, 1\right)^{2}+8 l^{3} x^{2}=0, \\
\left(a, h, k_{\chi} x, 1\right)^{2} x-\left(h, k, b_{\chi} x, 1\right)^{2}=0,
\end{gathered}
$$

the second of which is more simply written

$$
\left(a, h,-k,-i \gamma(x, 1)^{3}=0 .\right.
$$

Hence, restoring the factor $l^{3}$, and also to avoid fractions introducing the factor $8 a^{4}$, the resultant of the two equations is

$$
=8 l^{3} a^{4} \Pi\left\{8 l^{3} x^{2}+c\left(a, h, k_{\chi} x, 1\right)^{2}\left(h, k, b_{\zeta}(x, 1)^{2}\right\},\right.
$$

where $\Pi$ denotes the product of the factors corresponding to the three roots $x_{1}, x_{2}, x_{3}$ of the equation

$$
\left(a, h,-k,-b^{\gamma} x, 1\right)^{3}=0
$$

or what is the same thing,

$$
a x^{3}+h x^{2}-k x-b=0
$$

so that the symmetric functions are to be found from

$$
\Sigma x_{1}=-\frac{h}{a}, \Sigma x_{1} x_{2}=-\frac{k}{a}, x_{1} x_{2} x_{3}=\frac{b}{a} .
$$

The required discriminant is the foregoing resultant multiplied by $R$, or say by $c^{2} R^{2}$, that is the discriminant $Q_{10}$ is

$$
=c^{2} R^{2} \cdot 8 l^{3} a^{4} \Pi\left(8 l^{3} x^{2}+\Omega\right),
$$

if for shortness we write

$$
\Omega=\left(a, h, k \gamma(x, 1)^{2} \cdot\left(a, k, b_{\chi} x, 1\right)^{2},\right.
$$


and when the symmetric functions have been expressed in terms of the coefficients, the result is to be expressed as a function of $\square, \nabla, \Theta, R$ by means of the values

$$
\begin{aligned}
c^{2} \square & =a^{2} b^{2}+4 a k^{3}+4 b h^{3}-6 a d h k-3 h^{2} k^{2}, \\
\nabla & =-l^{2}, \\
c \Theta & =-l(a b-h k), \\
c R & =-8 l^{3} a b .
\end{aligned}
$$

Thus, for instance, the first term of the result is

which is

$$
=c^{2} R^{2} \cdot 8 l^{3} a^{4} \cdot 512 l^{3} x_{1}^{2} x_{2}^{2} x_{3}^{2},
$$

$$
\begin{aligned}
& =c^{2} R^{2} \cdot 4096 l^{12} a^{2} b^{2}, \\
& =c^{2} R^{2} \cdot-64 c^{2} R^{2} \nabla^{3}, \\
& =-64 c^{4} R^{4} \nabla^{3},
\end{aligned}
$$

which is a term in the before-mentioned expression for $Q_{10}$. 